\title{
SETDB1 prevents TET2-dependent activation of IAP retroelements in naïve embryonic stem cells
}

\author{
Özgen Deniz ${ }^{1}$, Lorenzo de la Rica ${ }^{1,2}$, Kevin C. L. Cheng ${ }^{1}$, Dominik Spensberger ${ }^{3,4}$ and Miguel R. Branco ${ }^{1 *}$
}

\begin{abstract}
Background: Endogenous retroviruses (ERVs), which are responsible for 10\% of spontaneous mouse mutations, are kept under control via several epigenetic mechanisms. The H3K9 histone methyltransferase SETDB1 is essential for ERV repression in embryonic stem cells (ESCS), with DNA methylation also playing an important role. It has been suggested that SETDB1 protects ERVs from TET-dependent DNA demethylation, but the relevance of this mechanism for ERV expression remains unclear. Moreover, previous studies have been performed in primed ESCS, which are not epigenetically or transcriptionally representative of preimplantation embryos.

Results: We use naive ESCS to investigate the role of SETDB1 in ERV regulation and its relationship with TETmediated DNA demethylation. Naïve ESCs show an increased dependency on SETDB1 for ERV silencing when compared to primed ESCs, including at the highly mutagenic intracisternal A particles (IAPs). We find that in the absence of SETDB1, TET2 activates IAP elements in a catalytic-dependent manner. Surprisingly, TET2 does not drive changes in DNA methylation levels at IAPs, suggesting that it regulates these retrotransposons indirectly. Instead, SETDB1 depletion leads to a TET2-dependent loss of H4R3me2s, which is indispensable for IAP silencing during epigenetic reprogramming.
\end{abstract}

Conclusions: Our results demonstrate a novel and unexpected role for SETDB1 in protecting IAPs from TET2-dependent histone arginine demethylation.

Keywords: Embryonic stem cells, Retrotransposons, Intracisternal A particles (IAPs), SETDB1, Ten-eleven translocation enzymes, DNA methylation, Histone arginine methylation

\section{Background}

Endogenous retroviruses (ERVs) are retroelements bearing long terminal repeats (LTRs) and constitute approximately $10 \%$ of the mouse genome [1]. Whilst most ERVs are inactive, a subset of these genetic parasites retain their transposition ability and therefore pose a threat to genome integrity [2]. Indeed, around 10\% of mouse spontaneous mutations arise as a direct result of ERV insertions [3] and insertional mutagenesis by ERVs is frequently associated with murine cancers $[4,5]$. Therefore, numerous transcriptional and post-transcriptional host mechanisms have evolved to suppress ERV activity. DNA methylation (or 5-methylcytosine $(5 \mathrm{mC})$ ) plays an essential role in ERV

\footnotetext{
*Correspondence: m.branco@qmul.ac.uk

'Blizard Institute, Barts and The London School of Medicine and Dentistry,

QMUL, London E1 2AT, UK

Full list of author information is available at the end of the article
}

repression in postimplantation embryos and male germ cells $[6,7]$. However, during early preimplantation and primordial germ cell (PGC) development, the genome undergoes genome-wide DNA demethylation [8-12] and additional mechanisms are required to ensure ERV silencing. Indeed, ERV silencing in embryonic stem cells (ESCs) is largely dependent on post-translational modification of histones, in particular methylation at H3K9. Removal of the H3K9me3 histone methyltransferase SETDB1 and its co-repressor, KRAB-associated protein (KAP1, also known as TRIM28), leads to significant activation of ERVs in ESCs [13-15] and PGCs [16]. Interestingly, 5mC and H3K9me3 regulate largely non-overlapping subsets of ERVs in ESCs, with the notable exception of intracisternal A particles (IAPs), whose silencing depends on the synergistic action of both epigenetic marks [14, 15]. 
IAP elements are relatively resistant to DNA demethylation during epigenetic reprogramming $[6,16-18]$, which is presumably a host defence mechanism against these highly mutagenic ERVs. Maintenance of methylation at IAP and imprinting control regions is driven by the G9a/GLP complex, which recruits de novo DNA methyltransferases (DNMTs) independent of its H3K9 methyltransferase activity [19-21]. On the other hand, H3K9me2-enriched regions are refractory to demethylation during epigenetic reprogramming [22] via recruitment of the DNMT1 chaperone NP95/UHRF1 [23, 24].

A role for $\mathrm{H} 3 \mathrm{~K} 9 \mathrm{me} 3$ in protecting ERVs from TETmediated DNA demethylation has also been proposed in ESCs [25]. TET enzymes oxidize $5 \mathrm{mC}$ into 5hydroxymethylcytosine $(5 \mathrm{hmC})$ and other oxidative derivatives as part of an active DNA demethylation pathway [26-28]. We have previously shown that TET1 binds to multiple retroelements in ESCs, and that both TET1 and TET2 help to maintain LINE-1 elements in a hypomethylated state [29]. At LTR elements such as IAPs, it has been shown that loss of SETDB1 enables TET1 binding, concomitant with an accumulation of $5 \mathrm{hmC}$ at these sites [25]. However, this resulted in only very subtle DNA methylation changes, and it remains unknown whether these alterations affect the expression of IAP elements and other ERVs. Moreover, TET enzymes may act on ERVs via non-catalytic pathways, similar to what we observed in LINE-1 elements [29]. Finally, previous studies were performed using primed ESCs grown under standard serumcontaining conditions, which are highly methylated and express high levels of the de novo methyltransferases DNMT3A and DNMT3B $[17,18]$. These conditions may counteract and mask the catalytic activity of TET enzymes at IAPs and other ERVs. Global DNA demethylation can be induced in vitro by growing ESCs under the so-called $2 \mathrm{i}$ conditions, which more closely resemble inner cell mass cells, driving a naïve pluripotent state $[17,30]$.

Here we investigated the role of SETDB1 in the regulation of ERVs in naïve ESCs and its relationship with TETmediated DNA demethylation. We find that SETDB1 has a markedly more prominent role in ERV silencing in naïve cells compared to primed cells, including at IAP elements. The catalytic activity of TET2 contributes to IAP activation upon SETDB1 depletion, but surprisingly this is not linked to DNA methylation changes at IAPs. We show that instead TET2 drives a loss of the repressive H4R3me2s mark at IAPs.

\section{Results}

\section{SETDB1 safeguards ERV silencing in naïve ESCs}

To investigate the role of SETDB1 in ERV silencing in naïve ESCs, we switched serum-grown (i.e., primed) E14 ESCs to $2 \mathrm{i}$ culture conditions. Using deep sequencing of PCR amplicons from oxidative bisulfite (oxBS)-treated
DNA [31, 32], we first confirmed that $5 \mathrm{mC}$ levels were substantially lower in naïve versus primed ESCs at multiple ERVs, including IAP elements (Fig. 1a). RLTR4/ MuLV elements were already hypomethylated in primed ESCs and showed only a small decrease in $5 \mathrm{mC}$ levels in naïve ESCs. $5 \mathrm{hmC}$ levels were generally low and similar between both culture conditions (Additional file 1: Figure S1a). In line with previous reports [33], transcript levels of these ERVs were not significantly higher in naïve ESCs compared to primed ESCs, suggesting that other mechanisms compensate for the loss of DNA methylation to protect the genome against the activity of ERVs (Additional file 1: Figure S1b).

To identify SETDB1 targets in naïve ESCs, we depleted SETDB1 by lentiviral delivery of shRNAs (Additional file 1: Figure S1c) and performed RNA-seq on three independent biological replicates. As SETDB1 is essential for ESC survival, we collected cells 5 days post-infection, before significant cell death is detected. For comparison, we conducted the same experiment in primed ESCs. Using an "inclusive mapping" strategy that harvests information from nonuniquely aligned reads (see "Methods"), we identified classes of repetitive elements that were differentially expressed by more than twofold $(p<0.05$, DESeq 2$)$. As expected, SETDB1 depletion in naive ESCs led to the upregulation of many repeat classes $(n=104)$, the vast majority of which were ERVs (Fig. 1b). Notably, about half of these repeat classes $(n=55)$ were exclusively upregulated in naïve cells and not in primed cells (Additional file 2). These naïve-specific repeats included MERVL, LINE-1 and VL30 elements, amongst several others. In contrast, only eight repeat classes were significantly upregulated exclusively in primed ESCs (Additional file 2).

Intriguingly, although IAP elements were deregulated in both culture conditions, they were substantially more activated in naïve ESCs when compared to primed ESCs (Fig. 1b; Additional file 2). We validated these observations using quantitative reverse transcription polymerase chain reaction (RT-qPCR), which confirmed that IAPEz upregulation was more pronounced in naïve cells (Fig. 1C), even though SETDB1 depletion led to a substantial loss of H3K9me3 at these elements in both conditions (Fig. 1d). Increased IAP upregulation in naïve ESCs was also confirmed in two additional ESC lines (Tet2 WT ESCs in Fig. 2c and Additional file 1: Figure S2e, and not shown). Similar results were obtained upon KAP1 depletion (Additional file 1: Figure S1d), as expected from the dependency of SETDB1 binding on $\operatorname{KAP1}[13,15]$. We then asked what fraction of IAP copies underwent this skewed IAP activation in SETDB1-depleted naïve cells. We analysed data from uniquely mapped RNA-seq reads and found that, out of 1009 IAPs with detectable RNA-seq signal, 681 (67\%) were more than tenfold upregulated upon SETDB1 depletion in naïve ESCs, whereas in primed cells 


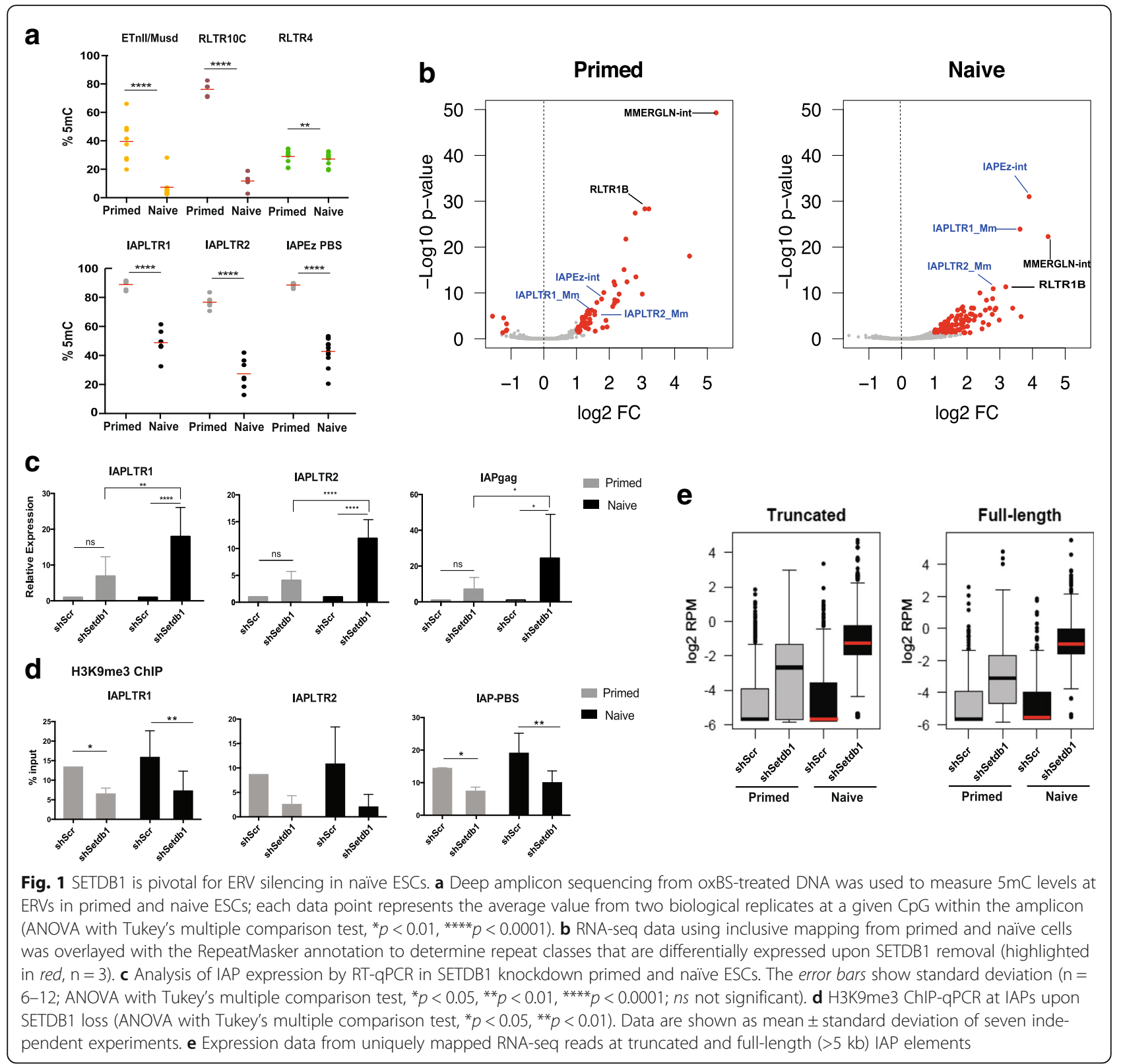

there were only 257 (25\%). Notably, this pattern was seen in both full-length and truncated elements (Fig. 1e), showing that SETDB1 removal leads to increased activation at the majority of mappable IAP elements in naïve ESCs compared to primed ESCs.

All together, these data show that SETDB1 plays a more prominent role in ERV silencing in naïve ESCs when compared to primed ESCs, including at IAP elements.

\section{IAP activation upon SETDB1 depletion depends on TET2 activity}

In naïve ESCs the role of SETDB1 in ERV suppression could be particularly critical for genome integrity due to the hypomethylated state of these retrotransposons.
Additionally, SETDB1 could protect ERVs from further DNA demethylation, by preventing binding of TET enzymes [25]. However, it remains unclear to what extent TET activity affects ERV methylation and expression. To address this question, we first performed ChIP-qPCR on wild-type (WT) and TET-depleted ESCs, which revealed that both TET1 and TET2 specifically bind IAP elements at the LTR and primer binding site (PBS; where KAP1 is recruited) regions in both primed and naïve ESCs (Fig. 2a). Notably, the enrichment of both TET enzymes at IAPs was similar to that seen at LINE-1 elements, which we have previously shown to undergo TET-mediated DNA demethylation [29]. To test whether TET enzymes were involved in the activation of IAPs, we performed 


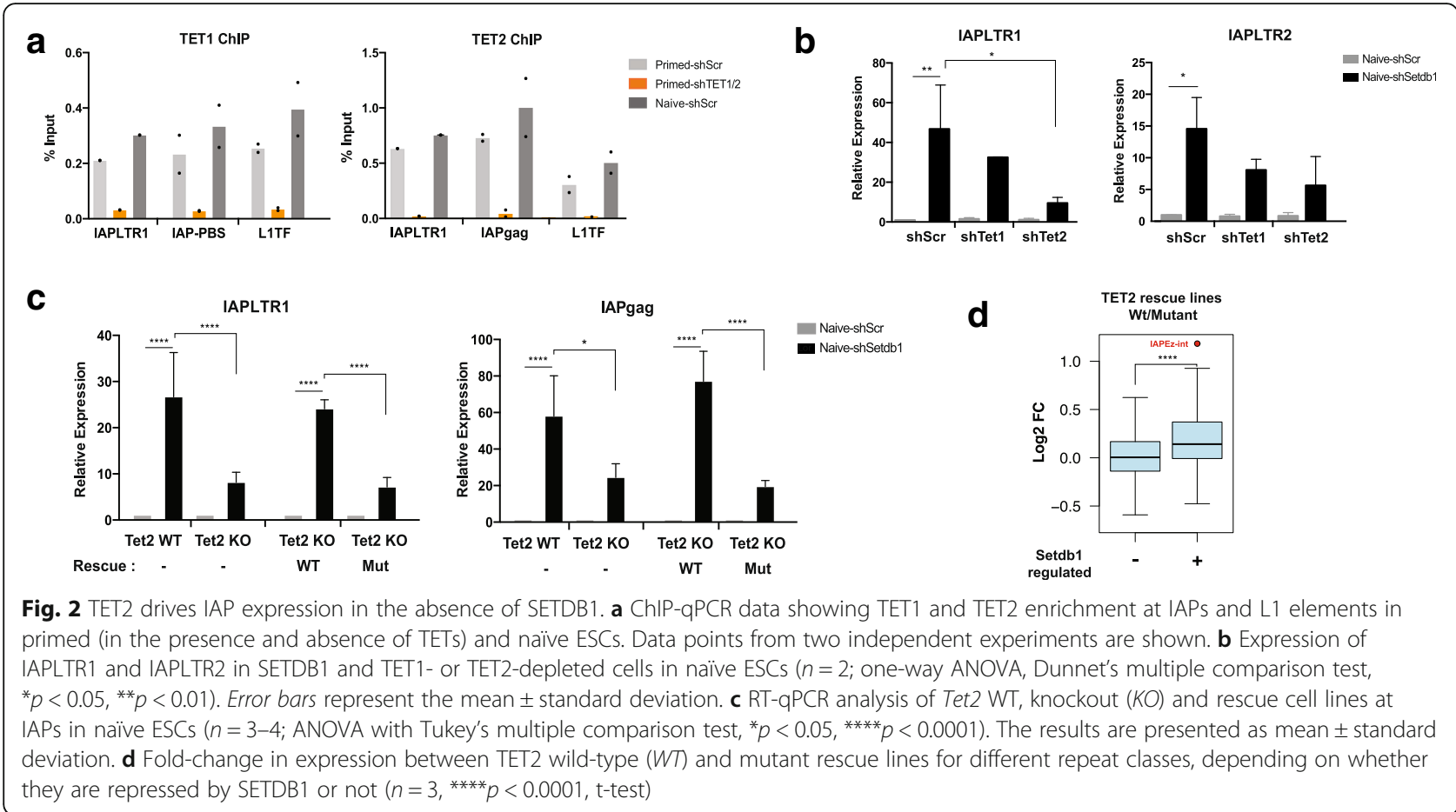

Tet1 or Tet2 knockdown (KD) in SETDB1-depleted naïve ESCs. Our RT-qPCR analyses revealed that removal of TET2 markedly reduced IAPLTR1 activation (and IAPLTR2 to a lesser extent) in SETDB1-depleted cells, whereas this effect was milder upon Tet1 KD (Fig. 2b). We also generated Tet2 knockout (KO) ESCs (Additional file 1: Figure S2a,b) wherein, similarly to Tet2 KD cells, upregulation of IAPLTR1, as well as the coding gag region, was diminished when compared to Tet2 WT naïve ESCs (Fig. 2c). Experiments involving depletion of both TET1 and TET2 showed that loss of TET2 alone was sufficient to maximally impair IAP activation (Additional file 1: Figure S2c).

We then asked whether the effect of TET2 is dependent on its $5 \mathrm{mC}$-oxidising catalytic activity. For this purpose, we used Tet $2 \mathrm{KO}$ ESCs to establish stable cell lines expressing either WT TET2 protein or a catalytically inactive mutant version of the enzyme. Western blot analyses revealed that both WT and mutant proteins were expressed at similar levels (Additional file 1: Figure S2d). Importantly, global $5 \mathrm{hmC}$ levels were similar between the parental WT ESCs and cells rescued with WT TET2, whereas both TET2 KO ESCs and cells rescued with mutant TET2 had low levels of $5 \mathrm{hmC}$ (Additional file 1: Figure S2d). Upon SETDB1 depletion, naïve ESCs expressing the WT Tet 2 construct displayed similar activation of IAPLTR1 and the gag region to what was seen in Tet2 WT cells, effectively rescuing the loss of TET2 (Fig. 2c). In contrast, cells expressing the catalytic mutant TET2 failed to upregulate IAPs any further than what was seen in SETDB1-depleted Tet2 KO cells (Fig. 2c). These results show that the catalytic activity of TET 2 contributes to the activation of IAPs upon SETDB1 depletion. To test whether other ERVs were targeted by the same mechanism, we performed RNA-seq on both TET2 rescue lines in a SETDB1-depleted context. Strikingly, comparison of ERV expression between WT and mutant TET2 rescue lines yielded only IAPEz elements as significantly differentially expressed. However, as a group, SETDB1-regulated repeats displayed higher expression levels in WT versus mutant TET2 rescue lines upon SETDB1 depletion, a tendency that was not seen at repeats that are not targeted by SETDB1 (Fig. 2d).

We also performed experiments in primed ESCs, wherein Tet2 $\mathrm{KO}$ had no effect on IAP upregulation upon SETDB1 depletion (Additional file 1: Figure S2). On the other hand, both RT-qPCR and RNA-seq data showed that overexpression of WT TET2 could also drive an increase in IAP activation in primed ESCs (Additional file 1: Figure S2e).

Overall, these results reveal that the activation of IAPs seen upon SETDB1 loss partly depends on the catalytic activity of TET2.

\section{SETDB1 does not protect IAPs from TET-mediated DNA demethylation}

The contribution of TET2 catalytic activity to IAP activation in SETDB1-depleted naive cells suggests that SETDB1 protects IAPs from oxidation-driven DNA demethylation. To directly address this hypothesis, we measured $5 \mathrm{mC}$ and 
$5 \mathrm{hmC}$ levels at IAPs using oxBS. Surprisingly, we did not observe any significant changes in $5 \mathrm{mC}$ levels in SETDB1depleted naive ESCs both at the LTR and PBS regions (Fig. 3a). IAP 5hmC levels remained low after SETDB1 depletion and only the PBS region displayed a small increase in $5 \mathrm{hmC}$ levels (Additional file 1: Figure S3a). In line with these observations, we found that TET2 binding to IAPs was not enhanced by the loss of SETDB1 in naïve ESCs (Fig. 3b). KAP1 depletion in naïve cells also did not lead to demethylation of IAPLTR1 elements, and was only associated with a small but significant decrease in IAPLTR2 methylation (Additional file 1: Figure S3b).

Extending our analyses to other SETDB1-regulated ERVs, we found that ETn/MusD, RLTR10C and VL30 elements also did not display a decrease in $5 \mathrm{mC}$ upon SETDB1 depletion in naïve ESCs (Fig. 3c). In contrast, primed ESCs displayed a small but significant loss of $5 \mathrm{mC}$ at IAPLTR2, ETnII/MusD and RLTR10C ERVs upon

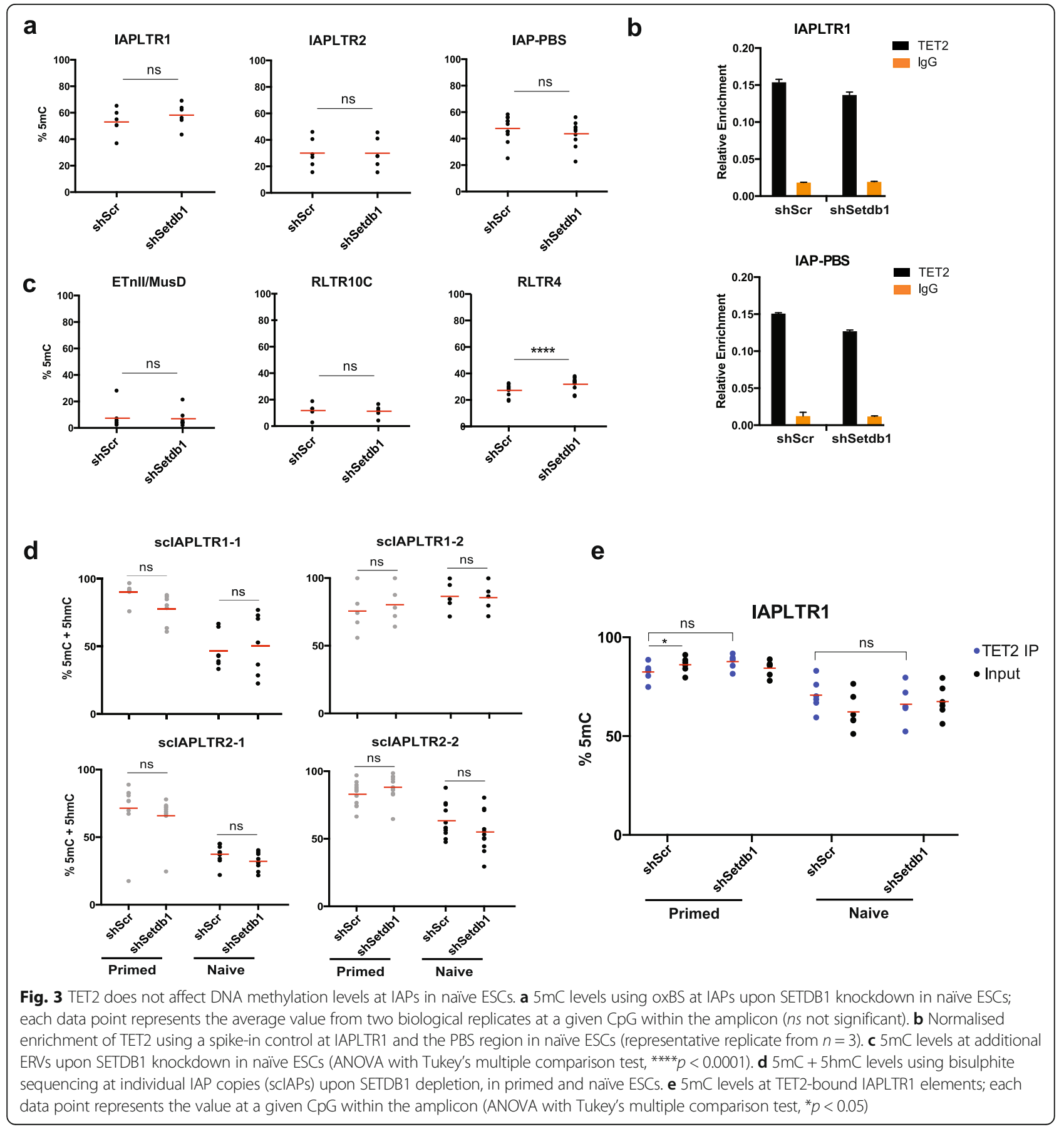


SETDB1 depletion, which is consistent with previous findings [13, 25] (Additional file 1: Figure S3c). However, these small reductions in $5 \mathrm{mC}$ levels were not associated with changes in expression, as IAPLTR2 transcripts are not affected in primed Tet $2 \mathrm{KO}$ cells (Additional file 1: Figure S2e). Furthermore, the lack of $5 \mathrm{mC}$ changes in naïve cells suggests that TET2 does not affect ERV expression by driving their demethylation.

We next considered the possibility that $5 \mathrm{mC}$ changes may be apparent in copies that are more responsive to SETDB1 depletion, rather than in the pool of IAP copies that are amplified by the consensus primers used above. Based on our RNA-seq data, we designed specific primers for bisulphite sequencing that target three individual IAPLTR1 and two individual IAPLTR2 elements that exhibited high activation upon SETDB1 depletion. Notably, these individual elements show higher levels of TET2 binding compared to a pool of IAP copies, whereas H3K9me3 and TET1 levels are similar (Additional file 1: Figure 3d). Despite displaying higher TET2 levels, the tested IAP copies did not show any significant alterations in DNA methylation in the absence of SETDB1 in either naïve or primed ESCs (Fig. 3d; Additional file 1: Figure S3e).

Vitamin $C$ is known to enhance the catalytic activity of TET enzymes, and it was recently shown to drive demethylation of IAP elements in naïve ESCs [33]. Indeed, we found that IAP methylation was lower in ESCs grown in the presence of vitamin $C$ and this was associated with an increase in IAP expression (Additional file 1: Figure S4a,b). However, SETDB1 depletion in cells treated with vitamin $\mathrm{C}$ had no additional effect on methylation levels (Additional file 1: Figure S4a), and the increase in IAP expression (relative to scrambled control) was similar to that seen in cells without vitamin C (Additional file 1: Figure S4b).

To confirm that TET2 did not modulate DNA methylation levels at IAPs, we performed bisulphite sequencing in TET2-depleted naïve cells. As expected, removal of TET2 did not affect methylation levels both for a pool of IAP copies as well as individual copies (Additional file 1: Figure S4c). Finally, we asked whether the effect of TET2 on DNA methylation is evident only at its target IAP copies. To this end, we analysed methylation levels of DNA that is immunoprecipitated by a TET2 antibody. DNA methylation levels of TET2-bound IAPs were comparable to the whole pool of IAPs (input) in all conditions, and no differences were seen between TET2-bound elements before or after SETDB1 depletion (Fig. 3e).

Taken together, these observations reveal that SETDB1 does not safeguard IAPs from TET-mediated DNA demethylation and that TET2 induces IAP activation by a DNA methylation-independent mechanism.
TET2 expression is associated with loss of H4R3me2 at IAPs We hypothesized that the contribution of TET2 to ERV activation in SETDB1-depleted naïve ESCs could be affecting key histone marks, possibly through an indirect mechanism. Therefore, we first asked whether TET2 could contribute to the loss of H3K9me3 seen upon SETDB1 depletion, but found that Tet 2 knockdown did not affect the levels of H3K9me3 in naïve ESCs (Fig. 4a). It was previously shown that TET enzymes can recruit O-GlcNac transferase (OGT) to chromatin in ESCs [34], which in turn targets H3K4 methyltransferase SET1/ COMPASS complex [35]. However, our ChIP-qPCR analyses demonstrated that TET2 depletion did not affect H3K4me3 levels at IAPs, which remained very low in all conditions tested (Fig. 4a). We also found no differences in the levels of the H3K27me3 repressive mark (Additional file 1: Figure S5a). IAP elements were also shown to be highly enriched for symmetrical arginine dimethylation at H4R3 (i.e., H4R3me2s) [36, 37]. Importantly, removal of the arginine methyltransferase PRMT5 leads to derepression of IAPs in PGCs and blastocysts [37], suggesting that H4R3me2s is a key repressive mark of IAPs during epigenetic reprogramming. Therefore, we next carried out ChIP-qPCR for H4R3me2s and found reduced levels of this mark in SETDB1-depleted ESCs (Fig. 4b). Strikingly, this loss at IAPs was driven by the action of TET2, as the levels of H4R3me2s remained stable upon SETDB1 removal in Tet2 KD cells (Fig. 4b). These results suggest that TET2 contributes to IAP activation in SETDB1-depleted ESCs by modulating the levels of the repressive H4R3me2s mark.

To test whether a similar mechanism could be responsible for the activation of other ERVs, we mined publicly available ChIP-seq data for H4R3me2s in naïve ESCs [36]. Using uniquely mapped reads, we identified repeat classes that are enriched for H4R3me2s peaks over a random control (Additional file 1: Figure S5b). Interestingly, H4R3me2s-enriched repeats were preferentially activated upon SETDB1 depletion when compared to non-enriched repeats (Fig. 4c). Using ChIP-qPCR we validated the enrichment of H4R3me2s on three selected SETDB1-regulated retrotransposons (RLTR4/MuLV, RLTR10C and L1Gf) and tested whether, similar to IAPs, SETDB1 depletion led to H4R3me2s loss at these sites. However, none of the tested elements displayed a significant reduction in H4R3me2s levels upon SETDB1 removal (Fig. 4d). This is in line with the fact that, unlike IAPs, the expression of these retrotransposons was not mediated by the catalytic activity of TET2 (Additional file 1: Figure S5c). These data suggest that TET2-mediated loss of H4R3me2s is specific to IAPs and drives their activation.

Notably, all of the retrotransposons analysed above displayed similar levels of TET2 enrichment (Fig. 4e), showing that TET2 binding is not sufficient to impart a 


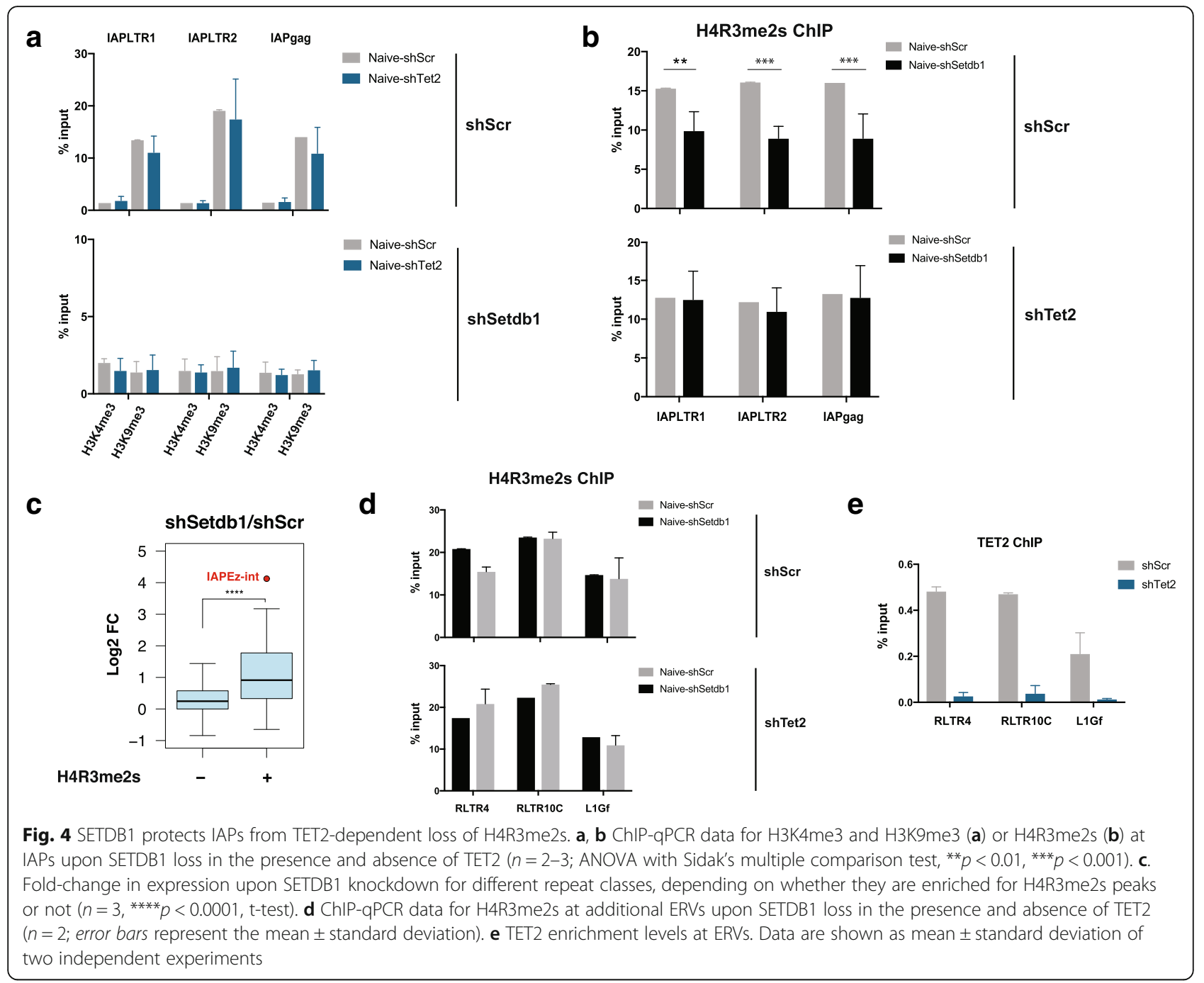

reduction in H4R3me2s levels. This suggest that TET2 activates IAPs in an indirect manner, possibly by regulating the expression of one or more chromatin modifiers that act on a subset of SETDB1-regulated retrotransposons.

\section{Discussion}

Here we have used naïve mouse ESCs to show that SETDB1 protects IAPs from TET2-dependent activation, but that instead of DNA demethylation this involves modulation of H4R3me2s levels at these elements.

We first established that SETDB1 has a more prominent role in ERV silencing in naïve ESCs than in primed ESCs. In contrast, in mouse embryonic fibroblasts ERV suppression is largely independent of SETDB1 [13], suggesting that cell differentiation is generally associated with a switch from an H3K9me3-dependent silencing mechanism to a $5 \mathrm{mC}$-dependent one. Our results suggest that such a reciprocal relationship extends further back into naïve pluripotency, where there is a more pronounced requirement for
SETDB1-mediated deposition of H3K9me3 for maintaining ERV silencing.

We show for the first time that the catalytic activity of TET2 contributes to IAP activation in SETDB1-depleted naïve ESCs. Unexpectedly, TET2 does not drive DNA demethylation at IAPs, including at individual IAP copies and at TET2-bound IAPs in naïve ESCs (Fig. 4). In contrast, a previous report suggested that in primed ESCs H3K9me3 deposition by SETDB1 protects IAPs from TET-mediated DNA demethylation [25]. Our data in primed ESCs partly support this (Additional file 1: Figure S3a), indicating that a potential direct relationship between SETDB1 and TET-mediated DNA demethylation is exclusive to the primed state and seemingly lost in naïve ESCs. Notably, even in primed ESCs, the extent of DNA demethylation is relatively small and is not associated with expression changes. In contrast, SETDB1 depletion has a major impact on the DNA methylation levels of germ line-specific genes and imprinting control regions (ICRs) [25]. In the case of ICRs, SETDB1 
presumably acts as a downstream effector of ZFP57 (via KAP1), which binds methylated ICRs to maintain allele-specific DNA methylation [38, 39].

We find that, rather than affecting IAP expression through changes in DNA methylation, TET2 drives a decrease in H4R3me2s levels at IAPs in naïve ESCs (Fig. 4b). It has been shown that in both PGCs and preimplantation embryos, deletion of Prmt5 leads to loss of H4R3me2s at IAPs, concomitant with their transcriptional activation [37]. Importantly, Prmt5-null PGCs display no differences in IAP DNA methylation levels compared to WT tissues [37]. Our data in naïve ESCs adds further support to a repressive role of the H4R3me2s mark at IAPs, as other key histone modifications were excluded as the mediators of TET2-dependent IAP activation (Fig. 4a; Additional file 1: Figure S5a).

Whilst the catalytic activity of TET2 does not affect DNA methylation directly at IAPs, it remains formally possible that TET2 oxidises methylated proteins or RNA associated with IAP chromatin. However, our data suggest that the action of TET2 on IAPs is likely to be indirect, involving the regulation of genes that in turn control IAP expression. Our RNA-seq data show that neither the arginine methyltransferases Prmt5 and Prmt7 nor the putative H4R3me2s demethylase Jmjd6 are controlled by TET2 activity (Additional file 1: Figure S5d). It remains to be tested whether other enzymes act in vivo to modify H4R3me2s, which could then mediate the activating effect of TET2 on IAPs. Other more indirect scenarios are also possible, such as a TET2-regulated gene that prevents recruitment of the enzymes involved in arginine methylation.

If TET2-mediated demethylation indirectly affects IAP expression, then the same could be true for other hypomethylation models, such as Dnmt KO ESCs. This raises questions about reported roles of DNA methylation on IAP expression, namely its synergistic action with H3K9me3 [14, 40]. Similarly, Tet1/Tet3 double KO preimplantation embryos display a downregulation of IAPs [41], but direct evidence for a causal relationship is missing. These considerations highlight the need for future work to harvest the power of epigenetic editing tools to test for direct causal links between ERV methylation and their activation.

\section{Conclusions}

We have demonstrated that SETDB1 has a more prominent role in ERV silencing in naive ESCs than primed ESCs, with the removal of SETDB1 leading to increased upregulation of ERVs. Our data show that activation of IAPs in SETDB1-depleted naive cells depends on the catalytic activity of TET2. However, surprisingly, TET2 does not play a role in DNA demethylation at IAPs; instead, TET2-dependent activation of IAPs is associated with the loss of the H4R3me2s repressive mark upon SETDB1 removal. In conclusion, our findings demonstrate a novel role of SETDB1 in protecting IAPs from TET2-dependent loss of H4R3me2s in naïve ESCs.

\section{Methods}

\section{Cell lines}

E14 ESCs were used for all experiments unless otherwise stated. Tet1 KO ESCs were a kind gift from Guo-Liang $\mathrm{Xu}$ [42]. Tet $2 \mathrm{KO}$ ESCs were generated by targeting exon 14 of Tet 2 with a floxed neomycin resistance cassette (Additional file 1: Figure S2a). To rescue the expression of TET2, stable cell lines were derived from Tet2 KO ESCs using a PiggyBac transposon system. The Tet2 catalytic mutant (H1304Y, D1306A) construct was made from a WT clone (a kind gift from Kristian Helin) by site-directed mutagenesis.

\section{Cell culture and gene knockdown}

ESCs were grown in feeder-free conditions using either DMEM-based medium with 15\% FBS and 1000 $\mathrm{U} / \mathrm{ml}$ ESGRO LIF (Millipore) or in 2i culturing conditions using DMEM F-12 (Gibco 21331) and neurobasal media (Gibco 21102) supplemented with N2 (Life tech. 17502048), B27 (Gibco 17504-044), $1000 \mathrm{U} / \mathrm{ml}$ ESGRO LIF (Millipore), Mek inhibitor (PD0325901) and GSK3b inhibitor (CHIR99021). For shRNA-mediated gene knockdown, ESCs were infected with viral particles carrying pLKO.1 constructs harbouring gene-specific shRNAs from The RNAi Consortium (shSETDB1, CCCG AGGCTTTGCTCTTAAAT, TRCN0000092975; shTET1, TTTCAACTCCGACGTAAATAT, TRCN0000341848; sh TET2, TTCGGAGGAGAAGGGTCATAA, TRCN000025 0894) or a non-targeting sequence (shScr, CCTAAGGTT AAGTCGCCCTCGCTC). After $48 \mathrm{~h}$, cells were selected with $1 \mu \mathrm{g} / \mathrm{ml}$ puromycin or $50 \mu \mathrm{g} / \mathrm{ml}$ hygromycin for 3 days.

\section{RNA isolation and RT-qPCR}

RNA was extracted using an AllPrep DNA/RNA mini kit (Qiagen 80204) and DNAse treated with the TURBO DNA-free $^{\mathrm{Tm}}$ Kit (Ambion, AM1907). RNA $(1 \mu \mathrm{g})$ was retrotranscribed using Revertaid Reverse Transcriptase (Thermo Scientific EP0441) and the cDNA was diluted 1/50 for qPCRs using MESA BLUE MasterMix (Eurogenentec, 10-SY2X-03 + NRWOUB) on a LightCycler 480 Instrument II (Roche). A list of primers used can be found in Additional file 3.

\section{RNA-seq library preparation}

For analysing the effects of SETDB1 depletion, ribosomal RNA-depleted RNA-seq libraries were prepared from 200-500 ng of total RNA using the low input ScriptSeq Complete Gold Kit (Epicentre). For the TET2 
rescue samples, mRNA libraries were prepared using the Dynabeads mRNA purification kit (Life Technologies) and the NEBnext Ultra RNA library prep kit (NEB). Libraries were sequenced on an Illumina NextSeq 500 with single-end 75-bp reads.

\section{Chromatin immunoprecipitation}

ChIP was performed as described in Latos et al. [43], with modifications. For the detection of TET1 and TET2, cells were fixed with an initial cross-linking step of 45 minutes with $2 \mathrm{mM} \mathrm{Di}(\mathrm{N}$-succinimidyl) glutarate (Sigma-Aldrich, catalogue number 80424) in PBS at room temperature, followed by a PBS wash and a second fixation step of 12 minutes with $1 \%$ formaldehyde in PBS. For histone ChIPs (H3K9me3, H3, H3K4me3, H4R3me2s) the cells were fixed with $1 \%$ formaldehyde for 12 minutes in PBS. After quenching with glycine, washes and lysis, chromatin was sonicated using a Bioruptor Pico from Diagenode, to an average size of 200700 bp. Immunoprecipitation was performed using $100 \mu \mathrm{g}$ of chromatin and $7.5 \mu \mathrm{g}$ of antibody (TET1, TET2) or $20 \mu \mathrm{g}$ of chromatin and $2.5 \mu \mathrm{g}$ of antibody (histones). Final DNA purification was performed using the GeneJET PCR Purification Kit (Thermo Scientific, catalogue number K0701) and elution in $80 \mu \mathrm{L}$ of elution buffer. This was diluted $1 / 10$ and analysed by qPCR, using the KAPA SYBR $^{\circ}$ FAST Roche LightCycler ${ }^{\circ} 480$ 2X qPCR Master Mix (Kapa Biosistems, catalogue number KK4611). A list of primers and antibodies used can be found in Additional files 3 and 4, respectively.

\section{Global $5 \mathrm{hmC}$ quantification}

Global levels of $5 \mathrm{hmC}$ were measured using the Global $5 \mathrm{hmC}$ quantification kit (Active Motif), following the manufacturer's instructions and using $25 \mathrm{ng}$ of DNA per well.

\section{Oxidative bisulphite sequencing}

Deep sequencing of PCR products from BS- and oxBSconverted DNA was performed as previously described [31]. Briefly, precipitated DNA (without glycogen) was resuspended in water and further purified using Micro Bio-Spin columns (Bio-Rad), after which half of the DNA was oxidised with $15 \mathrm{mM} \mathrm{KRuO}_{4}$ (Alpha Aesar) in $0.5 \mathrm{M} \mathrm{NaOH}$ for $1 \mathrm{~h}$. Following bisulphite conversion of both DNA fractions with the EpiTect Bisulfite kit (QIAGEN), a two-step PCR amplification was used: a first PCR amplifies the region of interest and adds part of the sequencing adaptors; a second PCR on pooled amplicons then completes the adaptors and adds sample barcodes, allowing for multiplexing (see primers in Additional file 3). Paired-end sequencing of pooled samples was done using an Illumina MiSeq.

\section{High-throughput sequencing data processing}

Reads were trimmed using Trim_galore! v0.3.3 with default parameters. External ChIP-seq data for H4R3me2s (GEO accession GSE37604) [36] were aligned to the $\mathrm{mm} 9$ genome assembly using Bowtie2 v2.1.0 [44] and uniquely aligned reads were extracted for peak detection using MACS2. To identify repeats enriched for H4R3me2s, the number of ChIP-seq peaks overlapping each repeat class were compared with a random control where peaks were shuffled (using bedtools) over mappable regions of the genome. RNA-seq data were aligned to $\mathrm{mm} 9$ using TopHat v2.0.9 [45] with -g 1 option, which assigns reads with multiple hits of equal quality to one of those locations at random (i.e., "inclusive mapping"). Raw read counts for each gene or Repeatmasker class were used in DESeq2 for differential expression analysis and for generating normalised gene and repeat expression values. For expression analysis of individual IAP copies, only uniquely mapped reads were used, together with a custom annotation of IAPs which merged same-strand IAP fragments within $100 \mathrm{bp}$ into a single element; elements longer than $5 \mathrm{~kb}$ were classified as full-length. Only elements with $>0.25$ RPM in any of the analysed samples were used. OxBS data were aligned with Bismark [46] to a custom genome containing the amplicon sequences; only CpGs covered by at least 100 reads were used to calculate $5 \mathrm{mC} / 5 \mathrm{hmC}$ levels.

\section{Additional files}

Additional file 1: Figures S1-S5. Figure S1. SETDB1 regulates ERV silencing in naïve ESCs. Figure S2. Removal of TET2 dampens SETDB1mediated IAP activation in naïve cells. Figure S3. SETDB1-mediated IAP activation is not linked to DNA methylation changes. Figure S4. SETDB1 depletion does not lead to DNA methylation changes. Figure S5. TET2 activity is associated with loss of H4R3me2s at IAPs. (PDF $828 \mathrm{~kb}$ )

Additional file 2: Table S1. List of differentially expressed repeat classes upon SETDB1 depletion. (XLSX 68 kb)

Additional file 3: Table S2. List of primers used in this study. (XLSX $52 \mathrm{~kb}$ ) Additional file 4: Table S3. List of antibodies used in this study. (XLSX $35 \mathrm{~kb}$ )

\section{Acknowledgements}

We thank the Barts Genome Centre for high-throughput sequencing, Guo-Liang Xu for the Tet1 KO ESCs, Tony Green for the Tet2 KO ESCs, Jesper Christensen and Kristian Helin for the anti-TET2 antibody and the Tet2 entry clone, and Paul Hurd and Vardhman Rakyan for critical reading of the manuscript.

\section{Funding}

M.R.B. is a Sir Henry Dale Fellow (101225/Z/13/Z), jointly funded by the Wellcome Trust and the Royal Society. Ö.D. and L.R. have received funding from the People Programme (Marie Curie Actions) of the European Union's Seventh Framework Programme (FP7/2007-2013) under REA grant agreement number 608765 .

\section{Availability of data and materials}

The datasets generated during the current study (RNA-seq) are available in the GEO repository under the accession number GSE100864. ChIP-seq data for H4R3me2s (GEO accession GSE37604) [36] were downloaded from the NCBI Gene Expression Omnibus. 


\section{Authors' contributions}

ÖD and MB designed the study and experiments. ÖD performed cell culture, shRNA knockdowns, RT-qPCR, western blotting, oxBS and ChIP experiments. LR performed ChIP experiments. KC performed QPCR analyses. DS generated the Tet2 KO ESCs. MB performed oxBS, RNA-seq and bioinformatic analyses. ÖD and MB wrote the manuscript with all other authors. All authors read and approved the final manuscript.

\section{Ethics approval and consent to participate}

Not applicable.

\section{Competing interests}

The authors declare that they have no competing interests.

\section{Publisher's Note}

Springer Nature remains neutral with regard to jurisdictional claims in published maps and institutional affiliations.

\section{Author details}

'Blizard Institute, Barts and The London School of Medicine and Dentistry, QMUL, London E1 2AT, UK. ${ }^{2}$ Present address: The Royal Society, 6-9 Carlton House Terrace, London SW1Y 5AG, UK. ${ }^{3}$ Department of Haematology, Cambridge Institute for Medical Research, University of Cambridge, Cambridge CB2 OXY, UK. ${ }^{4}$ Present address: Gene Targeting Facility, Babraham Institute, Cambridge CB22 3AT, UK

Received: 20 September 2017 Accepted: 7 December 2017

Published online: 19 January 2018

\section{References}

1. Waterston, et al. Initial sequencing and comparative analysis of the mouse genome. Nature. 2002;420:520-62.

2. Jern P, Coffin JM. Effects of retroviruses on host genome function. Annu Rev Genet. 2008:42:709-32.

3. Maksakova IA, Romanish MT, Gagnier L, Dunn CA, Van De Lagemaat LN, Mager DL. Retroviral elements and their hosts: insertional mutagenesis in the mouse germ line. PLoS Genet. 2006;2:e2.

4. Lee J, Haruna T, Ishimoto A, Honjo T. Intracisternal type A particle-mediated activation of the Notch4/int3 Gene in a mouse mammary tumor: generation of truncated Notch4/int3 mRNAs by retroviral splicing events. J Virol. 1999:73:5166-71.

5. Ukai $\mathrm{H}$, Ishii-oba $\mathrm{H}$, Ukai-tadenuma $\mathrm{M}$, Ogiu T, Tsuji H. Formation of an active form of the interleukin-2/15 receptor b-chain by insertion of the intracisternal A particle in a radiation-induced mouse thymic lymphoma and its role in tumorigenesis. Mol Carcinog. 2003;119:110-9.

6. Walsh C, Chaillet J, Bestor T. Transcription of IAP endogenous retroviruses is constrained by cytosine methylation. Nat Genet. 1998:20:116-7.

7. Bestor TH. Meiotic catastrophe and retrotransposon reactivation in male germ cells lacking Dnmt3L. Nature. 2004;431:2-5.

8. Kobayashi H, Sakurai T, Miura F, Imai M, Mochiduki K, Yanagisawa E, et al. High-resolution DNA methylome analysis of primordial germ cells identifies gender-specific reprogramming in mice. Genome Res. 2013;23:616-27.

9. Hajkova P, Ancelin K, Waldmann T, Lacoste N, Lange UC, Cesari F, et al. Chromatin dynamics during epigenetic reprogramming in the mouse germ line. Nature. 2008:452:877-81

10. De Felici M, Walsh CP. Methylation dynamics of repetitive DNA elements in the mouse germ cell lineage. Genomics. 2003;82:230-7.

11. Seisenberger S, Andrews S, Krueger F, Arand J, Walter J, Santos F, et al. The dynamics of genome-wide DNA methylation reprogramming in mouse primordial germ cells. Mol Cell. 2012;48:849-62.

12. Smith ZD, Chan MM, Mikkelsen TS, Gu H, Gnirke A, Regev A, et al. A unique regulatory phase of DNA methylation in the early mammalian embryo. Nature. 2012:484:339-44

13. Matsui $T$, Leung D, Miyashita $H$, Maksakova IA, Miyachi $H$, Kimura $H$, et al Proviral silencing in embryonic stem cells requires the histone methyltransferase ESET. Nature. 2010;464:927-31.

14. Karimi MM, Goyal P, Maksakova IA, Bilenky M, Leung D, Tang JX, et al. DNA methylation and SETDB1/H3K9me3 regulate predominantly distinct sets of genes, retroelements, and chimeric transcripts in mESCs. Cell Stem Cell. 2011:8:676-87.
15. Rowe HM, Jakobsson J, Mesnard D, Rougemont J, Reynard S, Aktas T, et al KAP1 controls endogenous retroviruses in embryonic stem cells. Nature. 2010;463:237-40.

16. Liu S, Brind'Amour J, Karimi MM, Shirane K, Bogutz A, Lefebvre L, et al. Setdb1 is required for germline development and silencing of H3K9me3marked endogenous retroviruses in primordial germ cells. Genes Dev. 2014; 28:2041-55.

17. Ficz G, Hore TA, Santos F, Lee HJ, Dean W, Arand J, et al. FGF signaling inhibition in ESCs drives rapid genome-wide demethylation to the epigenetic ground state of pluripotency. Cell Stem Cell. 2013;13:351-9.

18. Habibi E, Brinkman AB, Arand J, Kroeze LI, Kerstens HHD, Matarese F, et al. Whole-genome bisulfite sequencing of two distinct interconvertible DNA methylomes of mouse embryonic stem cells. Cell Stem Cell. 2013:13:360-9.

19. Dong KB, Maksakova IA, Mohn F, Leung D, Appanah R, Lee S, et al. DNA methylation in ES cells requires the lysine methyltransferase G9a but not its catalytic activity. EMBO J. 2008;27:2691-701.

20. Tachibana M, Matsumura Y, Fukuda M, Kimura H, Shinkai Y. G9a/GLP complexes independently mediate $\mathrm{H} 3 \mathrm{~K} 9$ and DNA methylation to silence transcription. EMBO J. 2008:27:2681-90.

21. Zhang T, Termanis A, Ozkan B, Bao XX, Culley J, de Lima AF, et al. G9a/GLP Complex maintains imprinted DNA methylation in embryonic stem cells. Cell Rep. 2016;15:77-85.

22. Von Meyenn F, lurlaro M, Habibi E, He C, Reik W, Stunnenberg HG. Impairment of DNA methylation maintenance is the main cause of global demethylation in naive embryonic stem cells. Mol Cell. 2016;62:848-61.

23. Rothbart SB, Krajewski K, Nady N, Tempel W, Xue S, Badeaux Al, et al. Association of UHRF1 with methylated H3K9 directs the maintenance of DNA methylation. Nat Struct Mol Biol. 2012;19:1155-60.

24. Liu X, Gao Q, Li P, Zhao Q, Zhang J, Li J, et al. UHRF1 targets DNMT1 for DNA methylation through cooperative binding of hemi-methylated DNA and methylated H3K9. Nat Commun. 2013;4:1563.

25. Leung D, Du T, Wagner U, Xie W, Lee AY, Goyal P, et al. Regulation of DNA methylation turnover at LTR retrotransposons and imprinted loci by the histone methyltransferase Setdb1. Proc Natl Acad Sci U S A. 2014:111:6690-5.

26. Tahiliani M, Koh KP, Shen Y, Pastor WA, Bandukwala H, Brudno Y, et al. Conversion of 5-methylcytosine to 5-hydroxymethylcytosine in mammalian DNA by MLL partner TET1. Science. 2009;324:930-5.

27. Hackett JA, Sengupta R, Zylicz JJ, Murakami K, Lee C, Down TA, et al. Germline DNA demethylation dynamics and imprint erasure through 5hydroxymethylcytosine. Science. 2013;339:448-52.

28. Branco MR, Ficz G, Reik W. Uncovering the role of 5-hydroxymethylcytosine in the epigenome. Nat Rev Genet. 2012:13:7-13.

29. de la Rica L, Deniz O, Cheng KCL, Todd CD, Cruz C, Houseley J, et al. TETdependent regulation of retrotransposable elements in mouse embryonic stem cells. Genome Biol, 2016:17:234

30. Leitch HG, McEwen KR, Turp A, Encheva V, Carroll T, Grabole N, et al. Naive pluripotency is associated with global DNA hypomethylation. Nat Struct Mol Biol. 2013;20:311-6.

31. de la Rica L, Stanley JS, Branco MR. Profiling DNA methylation and hydroxymethylation at retrotransposable elements. In: Garcia-Pérez JL, editor. Transposons and retrotransposons: methods and protocols. New York, NY: Springer New York; 2016. p. 387-401.

32. Booth MJ, Branco MR, Ficz G, Oxley D, Krueger F, Reik W, et al. Quantitative sequencing of 5-methylcytosine and 5-hydroxymethylcytosine at singlebase resolution. Science. 2012;336:934-7.

33. Walter M, Teissandier A, Pérez-Palacios R, Bourc'his D. An epigenetic switch ensures transposon repression upon dynamic loss of DNA methylation in embryonic stem cells. Elife. 2016;5:e11418.

34. Vella P, Scelfo A, Jammula S, Chiacchiera F, Williams K, Cuomo A, et al. Tet proteins connect the O-linked $\mathrm{N}$-acetylglucosamine transferase Ogt to chromatin in embryonic stem cells. Mol Cell. 2013;49:645-56.

35. Deplus R, Delatte B, Schwinn MK, Defrance M, Méndez J, Murphy N, et al. TET2 and TET3 regulate GICNAcylation and H3K4 methylation through OGT and SET1/COMPASS. EMBO J. 2013:32:645-55.

36. Girardot M, Hirasawa R, Kacem S, Fritsch L, Pontis J, Kota SK, et al. PRMT5mediated histone $\mathrm{H} 4$ arginine-3 symmetrical dimethylation marks chromatin at $\mathrm{G}+\mathrm{C}$-rich regions of the mouse genome. Nucleic Acids Res. 2014:42:235-48

37. Kim S, Günesdogan U, Zylicz JJ, Hackett JA, Cougot D, Bao S, et al. PRMT5 protects genomic integrity during global DNA demethylation in primordial germ cells and preimplantation embryos. Mol Cell. 2014;5:564-79. 
38. Li X, Ito M, Zhou F, Youngson N, Zuo X, Leder P, et al. A maternal-zygotic effect gene, Zfp57, maintains both maternal and paternal imprints. Dev Cell. 2008;15:547-57.

39. Quenneville S, Verde G, Corsinotti A, Kapopoulou A, Jakobsson J, Offner S, et al. In embryonic stem cells, ZFP57/KAP1 recognize a methylated hexanucleotide to affect chromatin and DNA methylation of imprinting control regions. Mol Cell. 2011;44:361-72.

40. Sharif J, Endo TA, Nakayama M, Karimi MM, Shimada M, Katsuyama K, et al. Activation of endogenous retroviruses in Dnmt1-/- ESCs involves disruption of SETDB1-mediated repression by NP95 binding to hemimethylated DNA. Cell Stem Cell. 2015;19:81-94.

41. Kang J, Lienhard M, Pastor WA, Chawla A, Novotny M, Tsagaratou A, et al. Simultaneous deletion of the methylcytosine oxidases Tet1 and Tet3 increases transcriptome variability in early embryogenesis. Proc Natl Acad Sci U S A. 2015;112:E4236-45.

42. Zhang RR, Cui QY, Murai K, Lim YC, Smith ZD, Jin S, et al. Tet1 regulates adult hippocampal neurogenesis and cognition. Cell Stem Cell. 2013;13: 237-45

43. Latos PA, Goncalves A, Oxley D, Mohammed H, Turro E, Hemberger M. Fgf and Esrrb integrate epigenetic and transcriptional networks that regulate self-renewal of trophoblast stem cells. Nat Commun. 2015;6:7776.

44. Langmead B, Salzberg SL. Fast gapped-read alignment with Bowtie 2. Nat Methods. 2012;9:357-9

45. Trapnell C, Pachter L, Salzberg SL. TopHat: discovering splice junctions with RNA-Seq. Bioinformatics. 2009;25:1105-11.

46. Krueger F, Andrews SR. Bismark: a flexible aligner and methylation caller for Bisulfite-Seq applications. Bioinformatics. 2011;27:1571-2.

\section{Submit your next manuscript to BioMed Central and we will help you at every step:}

- We accept pre-submission inquiries

- Our selector tool helps you to find the most relevant journal

- We provide round the clock customer support

- Convenient online submission

- Thorough peer review

- Inclusion in PubMed and all major indexing services

- Maximum visibility for your research

Submit your manuscript at www.biomedcentral.com/submit 